Bundesgesundheitsbl 2018 $\cdot 61: 771-777$ https://doi.org/10.1007/s00103-018-2755-6 Online publiziert: 5. Juni 2018

(c) Der/die Autor(en) 2018

CrossMark

\title{
Robert Jakob
}

Weltgesundheitsorganisation (WHO), Genf, Schweiz

\section{ICD-11 - Anpassung der ICD an das 21. Jahrhundert}

Großteil der globalen Gesundheitsressourcen zu verteilen.

$\mathrm{Zu}$ den Nutzern der ICD gehören

Die Internationale statistische Klassifikation der Krankheiten und verwandter Gesundheitsprobleme (ICD) ist der internationale Standard zur Erfassung, Berichterstattung und Gruppierung von Zuständen und Faktoren, die die Gesundheit beeinflussen. Sie enthält Kategorien für Krankheiten, gesundheitsbezogene Zustände und äußere Ursachen von Krankheit oder Tod. Zweck der ICD ist es, die systematische Erfassung, Analyse, Interpretation und den Vergleich von Mortalitäts- und Morbiditätsdaten national und international in zeitlicher und räumlicher Hinsicht zu ermöglichen. Die ICD dient dazu, Diagnosen von Krankheiten und anderen Gesundheitsproblemen in einen alphanumerischen Code zu übersetzen und die sprachunabhängige Speicherung und Analyse der Daten zu ermöglichen.

Die ICD wurde vor 150 Jahren als internationale Liste der Todesursachen eingeführt. Seitdem hat sie sich zu einem umfassenden Klassifizierungs- und Dokumentationssystem für alle Bereiche des Gesundheitssektors entwickelt. Die ICD-11 ist mit dem Ziel entworfen worden, ein nützliches Instrument für

- Mortalitätsstatistik,

- Morbiditätsstatistik,

- Epidemiologie,

- Prävention,

- Forschung,

- Vergütung,

- Qualitätssicherung und

- Patientensicherheit

zu schaffen. Sie wird in der Primärversorgung, in der Sekundärversorgung und in der Forschung eingesetzt. Darüber hinaus wird die ICD verwendet, um den
- Fachleute für das Management von Gesundheitsinformationen,

- Forscher,

- medizinische Dokumentare,

- Informatiker,

- Krankenschwestern,

- Ärzte,

- andere Gesundheitsversorger,

- Patienten,

- Programmierer,

- politische Entscheidungsträger,

- Statistiker,

- Versicherer

und viele mehr.

Die ICD ist eine Kernklassifikation der Familie der internationalen gesundheitsrelevanten Klassifikationen. Andere Kernklassifikationen sind die Internationale Klassifikation der Funktionsfähigkeit, Behinderung und Gesundheit (ICF) und die Internationale Klassifikation der Gesundheitsinterventionen (ICHI), die sich in den letzten Schritten der Entwicklung befindet.

Die ICD wird je nach Bedarf in unterschiedlichen Auflösungsstufen eingesetzt, die im Falle der ICD-10 von ca. 100 Codes [1] über 2048 dreistellige Codes (sog. Dreisteller) bis zu mehr als 60.000 Codes (mit Ergänzungen; [2]) reichen. Die internationale Berichterstattung findet allerdings mindestens aufdem Niveau der Dreisteller statt (z. B. A00, „Cholera“).

Die elfte Revision der Internationalen Klassifikation der Krankheiten (ICD) ist ein großer Schritt vorwärts, dank der eingesetzten Methodik, Technik und ihrer wissenschaftlichen Überarbeitung. Die ICD-11 wurde allerdings mit Rücksicht auf frühere Revisionen der ICD entwickelt, um Zeitreihen für wichtige Erkrankungen oder Erkrankungsgruppen weiterhin zu ermöglichen.

Der Artikel gibt einen Einblick in die Klassifikationsentwicklung im 21. Jahrhundert am Beispiel der ICD-11, in Hinsicht auf Beteiligung, Transparenz des Prozesses, eingesetzter Technologien und Benutzung der ICD.

\section{Gründe für die Revision der ICD}

Ausgangspunkt für die Revision war zunächst eine Zusammenstellung der inhaltlichen und technischen Unzulänglichkeiten der ICD-10 (siehe - Tab. 1). Von inhaltlicher Seite haben die Bedürfnisse, spezielle Inhalte detaillierter verschlüsseln zu können, ihren Niederschlag in den verschiedenen nationalen klinischen Modifikationen der ICD gefunden [3]. Strukturell und inhaltlich war die ICD-10 durch einen Aktualisierungsmechanismus so weit wie möglich regelmäBig aktualisiert worden. Allerdings waren dem enge Grenzen im Rahmen der existierenden Struktur gesetzt, sodass die Mortalitäts- und die Morbiditätsarbeitsgruppe des Netzwerks der WHO-Kooperationszentren für die Klassifikationen eine Liste von Änderungswünschen zur Berücksichtigung in der ICD-11 erarbeiteten.

Auf technologischer Seite war es notwendig, die ICD von einem Buchprodukt in eine elektronische Infrastruktur zu überführen. Bei der ICD-10 erfolgen Pflege von Index und Systematik separat und in einer im Wesentlichen auf Buchdruck ausgelegten XML-Variante. Übersetzungen erfolgen in vielen unterschiedlichen Formaten und können nur schwer abgeglichen werden. 


\section{Tab. 1 Übersicht über die wesentlichen Gründe für die Revision}

\begin{tabular}{l|l} 
Wissenschaftliche Aktualisierung notwendig \\
\hline $\begin{array}{l}\text { Technische Umstellung auf } \\
\text { elektronische ontologische } \\
\text { Infrastruktur zur }\end{array}$ & $\begin{array}{l}\text { Eignung für elektronische Dokumentation } \\
\text { Verbesserung der Benutzerführung }\end{array}$ \\
& $\begin{array}{l}\text { Verbesserung der Abbildbarkeit relevanter Details } \\
\text { Unterstützung mehrsprachiger Anwendungen und Überset- } \\
\text { zungen } \\
\text { Vereinfachung der Pflege der Klassifikation }\end{array}$ \\
Anbindung an andere Terminologiesysteme
\end{tabular}
$\begin{aligned} & \text { Anpassung an die De-facto-Anwendungsfälle } \\
& \text { Einbindung von mehr Definitionen }\end{aligned}$

Ein weiterer wichtiger Aspekt bei der Revision war die Nutzerfreundlichkeit. Korrektes Codieren mit der ICD-10 erfordert die korrekte Nutzung des Bandes 3 (Index), des Bandes 1 (Systematisches Verzeichnis) und Kenntnis der Regeln und Konventionen in Bd. 2 (Regelwerk). Zur Erzielung einer guten Codierungsqualität mit der ICD-10 bedarf es eines erheblichen Trainingsaufwandes. Im Design der ICD-11 sind der Index, das Systematische Verzeichnis und die Codierregeln in eine einzige logische Struktur eingebunden. Sie wird „Foundation Component" genannt und ist weiter unten in einem eigenen Abschnitt beschrieben. Die auf dieser Struktur basierende Software kann die korrekte Nutzung der Klassifikation ohne wesentliches Training sicherstellen.

In etlichen Bereichen war auch der Verwendungszweck einzelner Kategorien der ICD nicht vollständig klar und es fehlten Definitionen, Beschreibungen und zusätzliche Hinweise, um die Nutzer zu führen [4].

Der Einzug der elektronischen Dokumentation in allen Bereichen des Gesundheitssektors und in allen Arbeitsumgebungen, einschließlich Bereichen mit begrenzten Ressourcen, war eine treibende Kraft zur strukturellen Umgestaltung der ICD von einem Buch zu einer Klassifikation auf ontologischer Basis in einer elektronischen Plattform.

\section{Strukturierte Einbindung internationaler Expertise durch weltweite Vernetzung}

Ein innovativer Aspekt bei der Organisation der Revision ist der Einsatz von
Onlineplattformen. Neben einer speziellen Autorenplattform [5], auf der die ICD bearbeitet wird, besteht auch eine Vorschlags- und Kommentierungsplattform, auf der, nach einfacher Selbstregistrierung, jede Person Beiträge zur Revision und nun auch zur weiteren Pflege leisten kann. Der Einsatz dieser Technologie hat eine viel breitere Beteiligung als bei allen vorangegangenen Revisionen ermöglicht. Insgesamt haben mehr als 297 Spezialisten von 260 Institutionen in 55 Ländern aller WHO-Regionen (Amerika, Ostmittelmeer und Nordafrika, Afrika, Europa, Südostasien, Westpazifik) im Rahmen der verschiedenen Themenbeiräte (Topic Advisory Groups) zu ICD-11 an der Überarbeitung mitgewirkt. Zusätzlich sind mehr als 10.000 Vorschläge aus 35 Ländern eingegangen, die, bis auf wenige Ausnahmen (die sich noch in Begutachtung befinden), alle bearbeitet wurden.

Erste Schritte zur Planung erfolgten ab 2001. Im Jahr 2006 wurde schließlich ein Projektplan erstellt, der Ziele, Methodik, Technik und den Zeitplan beschreibt [6, 7].

Die Überarbeitung von ICD-11 erfolgte in mehreren Phasen. Nach dem offiziellen Start der Revision in 2007 unterstützte zunächst eine Revisionslenkungsgruppe (Revision Steering Group) die WHO bei der Bildung von Themenberatungsgruppen (Topic Advisory Groups, TAG), der Planung und in wissenschaftlichen Fragen. Die Beratungsgruppen wurden in Zusammenarbeit mit internationalen wissenschaftlichen Gesellschaften und verschiedenen Abteilungen der WHO zusammengestellt und finanziell wie auch logistisch unterstützt.
Die Gruppen setzten sich aus Experten aus verschiedenen Regionen und Anwendungsbereichen der ICD zusammen. Ein Teil der Gruppen bearbeitete die ICD aus dem Blickwinkel eines bestimmten Fachbereichs. Diese Gruppen waren jeweils für bestimmte Kapitel verantwortlich. Dies beinhaltete auch, dass solch eine Gruppe eine Einigung im Falle von Konflikten in Überlappungsbereichen mit anderen Disziplinen herbeiführen musste. Andere Arbeitsgruppen, wie die für Pädiatrie und seltene Erkrankungen [8], arbeiteten horizontal durch alle Kapitel. Aufgrund der engen anatomischen und funktionalen Beziehungen wurden der Urogenitalbereich, das Kapitel „Schwangerschaft, Geburt und Wochenbett“ und das Kapitel „Bestimmte Zustände, die ihren Ursprung in der Perinatalperiode haben“, von einer einzigen Gruppe bearbeitet. Aus den gleichen Gründen wurden auch die Kapitel zu den Verletzungsfolgen und den äußeren Ursachen (ICD-10 XIX und XX) in einer Gruppe zusammengefasst. Eine spezielle Arbeitsgruppe mit Experten aus China, Japan und Korea erarbeitete das neue Kapitel „Traditionelle Medizin, Modul 1“.

Anwendungsfallbezogene Gruppen sorgten für Konsistenz der Klassifikation und überarbeiteten auch die jeweiligen Codierregeln. Eine Gruppe für Qualität und Patientensicherheit erstellte einen neuen Abschnitt im Kapitel „Äußere Ursachen“. Der andere Teil der Gruppen von Anwendern sorgte dafür, dass die Konsistenz der ICDs für die jeweilige Anwendung, auf die sie sich konzentrierten, gewährleistet war. Eine Beratergruppe für Gesundheitsinformationsmanagement unterstützte die WHO bei der Gestaltung der modernen ontologischen Struktur und dem Aufbau der dazugehörigen Informationstechnologie.

In der Phase 2(2015-2018), nach einer Begutachtung des Fortschrittes der Revision [9], wurde, mit Unterstützung einer neu gegründeten Beratergruppe aus dem Anwenderbereich, der Schwerpunkt auf die Konsolidierung der Klassifikation gelegt. Ziel war es, auf der Basis der Beiträge der wissenschaftlichen Arbeitsgruppen eine konsistente Gesamtstruktur zur Nutzung in der Dokumentation und Sta- 
tistik von Mortalität und Morbidität zu erstellen. Die wissenschaftlichen Arbeitsgruppen wurden in externe Beratergremien umgewandelt. Eine einzelne medizinisch-wissenschaftliche Beratergruppe (Medical Scientific Advisory Committee, MSAC) beantwortet nun wissenschaftliche Fragen unter bedarfsweiser Inanspruchnahme fachspezifischer Gruppen.

In der Revision zur ICD-11 wurden erstmalig Feldversuche vor ihrer Veröffentlichung durchgeführt. Eine spezielle Onlineplattform (Field Implementation and Test Platform, FIT) erlaubt das Testen von Codierverhalten und Fallbeispielen. Ein Vergleich mit den korrekten Ergebnissen ermöglicht auch Codiertests zur Überprüfung, inwieweit Übersetzungen mit dem englischen Original übereinstimmen. Einzelne Nutzer oder Nutzergruppen können sich auch zukünftig ihr Gesamtresultat ausgeben lassen.

Insgesamt haben in der Haupttestphase 1673 Teilnehmer aus 31 Ländern 112.383 Verschlüsselungen durchgeführt, darunter auch ein Feldtest in Deutschland Anfang 2017 in Zusammenarbeit zwischen dem Deutschen Institut für Medizinische Dokumentation und Information (DIMDI), der Arbeitsgruppe ICD des Kuratoriums für Fragen der Klassifikation im Gesundheitswesen und der Arbeitsgemeinschaft der Wissenschaftlichen Medizinischen Fachgesellschaften e.V. (AWMF). Die Tests gaben insbesondere Hinweise auf notwendige Verbesserungen in der Benutzerführung der ICD-11.

In dieser Phase 2 sind auch Stellungnahmen der WHO-Mitgliedsstaaten und Überprüfungen diverser Fachgesellschaften eingeflossen.

Die Phase 3 schließt sich an die Veröffentlichung der Implementierungsfassung der ICD-11 im Juni 2018 an. Sie dient der Vorbereitung zur Implementierung durch die Länder. Die Codestruktur bleibt ab dieser Fassung unverändert. Es wird erwartet, dass insbesondere die Übersetzungsarbeiten Hinweise auf die Notwendigkeit $\mathrm{zu}$ einzelnen Verbesserungen der Nutzerführung geben werden. Strukturelle Änderungswünsche werden dann ausschließlich im Rahmen des normalen Pflegemechanismus bearbeitet, in dem

Bundesgesundheitsbl 2018 $\cdot 61: 771-777$ https://doi.org/10.1007/s00103-018-2755-6 (c) Der/die Autor(en) 2018

\section{R. Jakob}

\section{ICD-11 - Anpassung der ICD an das 21. Jahrhundert}

\section{Zusammenfassung}

Die ICD-11 adressiert neben den medizinischen Neuerungen auch die veränderten Bedürfnisse der digitalisierten Gesundheitssysteme. In diesem Artikel sollen das Konzept und die Entwicklungsstruktur der ICD-11 dargestellt werden.

Die neue technische Struktur der ICD-11 erlaubt eine erheblich vereinfachte Nutzung der Klassifikation bei gleichzeitig deutlich verbesserter Fähigkeit, einzelne Gesundheitszustände im gewünschten Detailgrad zu dokumentieren. Die Integrierung der gesamten inhaltlichen und strukturellen Information der ICD-11 in einer ontologisch organisierten allumfassenden Gesamtstruktur, der sogenannten Foundation, ist in Kombination mit den angebundenen Onlineplattformen ein Schritt in die Zukunft: Übersetzung und Pflege der Klassifikation werden vereinfacht und die Konsistenz - insbesondere zwischen den verschiedenen Sprachfassungen erheblich verbessert. Die Foundation erlaubt auch die Ableitung anwendungsspezifischer Fassungen, sogenannter Linearisationen, die verschiedenen Nutzergruppen die Anwendung der ICD-11 erleichtern. Die ICD-11 erlaubt eine versionierungssichere Dokumentation unter Verwendung einer eineindeutigen Zeichenkette, dem Unique Reference Identifier (URI). Sie ist für die De-facto-Anwendungsfälle der Gegenwart entworfen worden. Die ICD-11 ist inhaltlich erneuert worden und angebunden an andere Klassifikationen und Terminologien. Diese Neuerungen ermöglichen eine zeitgemäße Dokumentation relevanter Gesundheitsinformation in allen Bereichen, die Zusammenführung von Daten und die Untersuchung von Zusammenhängen im Gesundheitsbereich.

\section{Schlüsselwörter}

Klassifikation · Diagnosen - ICD · Krankheiten · Morbidität · Mortalität

\section{ICD-11-Adapting ICD to the 21st century}

\section{Abstract}

In addition to medical innovations, the International Classification of Diseases (ICD11) addresses the changing needs of digitized healthcare systems. This article describes the concept and developmental structure of the ICD-11.

The new technical structure of the ICD11 allows for a considerably simplified use of the classification and at the same time significantly improves the ability to record individual health conditions at the desired level of detail. The integration of the entire contents and structural information of the ICD-11 in an ontologically organized allencompassing structure, the so called "foundation", in combination with the connected online platforms is a step into the future. It simplifies translation and maintenance of the classification and considerably improves consistency, especially between language versions. The foundation also permits the production of use-specific versions, so called linearisations, that facilitate the application of ICD by the relevant user groups. The ICD-11 allows versioning-safe documentation using a unique character string, the unique reference identifier. The ICD-11 has been designed for the current de facto use cases. The content of ICD-11 has been updated and linked to other classifications and terminologies. These innovations enable up-to-date documentation of relevant health information in all areas, aggregation of data and the analysis of examination of interrelationships in health.

\section{Keywords} Classification - Diagnosis - ICD · Diseases . Morbidity $\cdot$ Mortality das Classifications and Statistics Advisory Committee (CSAC) die Ergebnisse der Begutachtung von Änderungsvorschlägen durch das MSAC und verschiedener anwendungsfallbezogener Arbeitsgruppen in die Entscheidungsfindung zur Umsetzung eines Vorschlages einbe- zieht [10]. In diese Phase fällt auch die Verabschiedung der ICD-11 durch die Weltgesundheitsversammlung im Mai 2019 mit Inkrafttreten zum 1. Januar 2022. 


\section{Überleitungen}

Eine Mapping-Plattform befindet sich in einem nichtöffentlichen Bereich des ICD-11-Onlinebrowsers [11]. Diese Plattform unterstützt die manuelle Erstellung von Überleitungstabellen zwischen ICD-10 und ICD-11. Die Verknüpfungen erfolgen zwischen den korrespondierenden Einträgen der ICD-10 und der ICD11. Hierbei ist zu berücksichtigen, dass in der ICD-11 die logische hierarchische Struktur nicht mit der Ebene der Codierung endet, sondern bis zu 12 Verzweigungsebenen, wie Wurzeln, von Kapitelebene bis in die Tiefe reicht. Die Mapping-Plattform berechnet aus den manuell erstellten detaillierten Verbindungen dann die Überleitung auf der Ebene der Schlüsselnummern separat für beide Richtungen der Überleitung. Auch wird eine Version zur Umverschlüsselung ausgegeben, wie auch eine Tabelle mit den detaillierten Entsprechungen. Die fertigen Überleitungen können im Onlinebrowser heruntergeladen werden.

\section{Übersetzungen}

Der Onlinebrowser enthält auch eine Übersetzungsplattform. Diese erlaubt Übersetzungen auf Konzeptebene. Nach Bedarf können für jede Sprache unabhängig

- englische Vorzugsbegriffe für Konzepte und deren englische Synonyme übersetzt werden,

- sprachabhängige Synonyme hinzugefügt werden oder

- nur auf Englisch existierende Synonyme ausgelassen werden oder

- ein anderer Vorzugsbegriff für die Verwendung in den Klassentiteln einzeln angegeben werden.

Bei neuen Übersetzungen kann schnell überprüft werden, ob der Begriffoder Teile davon bereits übersetzt wurden. Dies erhöht die Konsistenz von Übersetzungen. Die Plattform ermöglicht einen Arbeitsablauf mit drei hierarchischen Ebenen und unterschiedlichen Zugriffsrechten.

Die Plattform ist schon seit dem Jahr 2016 in aktivem Gebrauch. Die spanische Übersetzung ist nahezu fertig; die chine- sische Übersetzung ist weit fortgeschritten und mehrere andere Sprachversionen befinden sich teils in der Übersetzung, teils in der Vorbereitung.

\section{Anwendung}

Die Codierung mit der ICD-11 erfolgt mithilfe des „Coding-Tools“ ([12], - Abb. 1), einem webbasierten Codierwerkzeug. Dieses Codierwerkzeug ersetzt den früheren Index als Referenz in Codierfragen. Die Codieranfragen liefern bei einer Suche nach sehr detaillierten Angaben auch die Sequenz an Schlüsselnummern, die zur vollständigen Beschreibung notwendig ist. Dazu wird auf die entsprechenden Einträge zur Zusatzverschlüsselung einzelner Kategorien zurückgegriffen. Das bedeutet eine erhebliche Vereinfachung der Nutzung der ICD-11 und reduziert den Trainingsaufwand für Codierer im Vergleich zur ICD-10 drastisch. Die Suchmaschine greift stets auf die sprachunabhängige (interne) logische Darstellung der ICD zurück. Somit kann sie, nach entsprechender Konfiguration in jeder Sprache, in die die ICD übersetzt ist, verwendet werden.

Die gleiche Funktionalität steht auch als Webservice zur Einbindung in eine beliebige Software zur Verfügung. Der Betrieb in der Cloud erlaubt eine Anpassung der Leistungsfähigkeit an die Zahl der Zugriffe und die Fortsetzung des Betriebs auch bei Ausfall einzelner Server oder Rechenzentren. Eine Offlineversion ist in Planung, wie auch Applikationen zum Einsatz auf Mobilgeräten.

\section{Das Fundament}

Die in den vorangegangenen Abschnitten beschriebene Funktionalität wird durch die sogenannte Foundation Component der ICD-11 ermöglicht. Alle Einträge (Kategorien, Entitäten, Gruppen, Synonyme und Exklusiva - detaillierte Beschreibungen finden sich in der sogenannten ICD-11 Reference Guide [13]) befinden sich in einer elektronischen Struktur. Hier werden die Beziehungen aller Konzepte zueinander in maschinenlesbarer Form beschrieben. Zum Beispiel ist dort angegeben, dass eine maligne
Neoplasie des Oesophagus ein Tumor ist, aber rein semantisch auch eine Erkrankung des Verdauungstraktes. Es ist dort allerdings auch angegeben, dass für die Zwecke der ICD-11-Klassifikation Fälle mit diesem Tumor zu den Tumorerkrankungen gezählt werden. Die zweite Verbindung zu den Erkrankungen des Verdauungstraktes zeigt sich dem Nutzer als Querverweis zu den Tumoren. Des Weiteren enthält die Foundation Component Beschreibungen der einzelnen Einträge in Bezug auf Anatomie, Ätiologie, diagnostische Kriterien und vieles mehr, das im Projektplan näher beschrieben ist. Hier erfolgt auch idealerweise die Anbindung externer Terminologien, wie die Systematized Nomenclature of Medicine-Clinical Terms (SNOMED CT). Dadurch wird eine identische und semantisch korrekte Beschreibung der Einträge der ICD an den Übergängen von solchen Terminologiesystemen gewährleistet. Ein Abgleich zwischen SNOMED CT und ICD-11 im Bereich der Erkrankungen des Kreislaufsystems und die Formulierung einer Überleitung von SNOMED CT zur ICD-10 haben gezeigt, dass eine semantische Annäherung zwischen SNOMED CT und ICD möglich ist. Gleichzeitig wurde aber klar, dass insbesondere der automatischen Überleitung von SNOMED CT zur ICD auch Grenzen gesetzt sind [14-18].

Im Fundament befinden sich auch Codierhinweise und die Angaben zu möglichen Kombinationen von Schlüsselnummern. All diese Informationen kommen beim Codierwerkzeug zum Einsatz und werden in benutzerfreundlicher Weise im Onlinebrowser der ICD-11 dargestellt. Das System erlaubt die Angabe von speziellen Konfigurationen für unterschiedliche Fassungen der ICD. Da alle Einträge in der ICD-11 - unabhängig von ihrem Dasein als Kategorie in der Klassifikation - eine eigene unveränderliche eineindeutige Zeichenkette, den Unique Reference Identifier (URI), haben, ist bei elektronischer Dokumentation des URI zusätzlich zur Schlüsselnummer eine eindeutige und zeitunabhängige Definition der Daten auch zwischen den verschiedenen Versionen gewährleistet.

Gegenwärtig sind Fassungen zur Codierung in der Primärversorgung in Um- 
arterial |

Related words...

Word list

sort: Relatedness/repetition

multiple

aneurysm

occlusion

embolism

congenital

occluded

coronary

vessel

cerebral

vein

thrombosis

ruptured

peripheral

pulmonary

infarction

type

thrombotic

precerebral

aorta

brain

injury

stenosis

disease

stricture

syndrome

carotid

vertebral

aortic

acquired

\section{$\mathbf{x}$}

The results shown are incomplete

Destination Entities

sort: Matching score

Chapter distribution / filter

BA2Z Hypotension, unspecified ${ }^{3}$ arterial hypotension :

BD4Z Chronic arterial occlusive disease, unspecified :

BD52.1 Arteriovenous fistula, acquired c] arterial varix

BD52.2 Stricture of artery $\equiv$ CU

BD52.Z Certain specified disorders of arteries or arterioles, unspecified $\equiv \mathrm{CU}^{\mathrm{C}}$

ND56.5 Injury of blood vessel of unspecified body region C" Injury of artery NOS :

BD52.3 Rupture of artery $\equiv \mathbb{E}^{2}$

MC9YOther specified symptoms or signs involving the circulatory system Ct Arterial murmur

MG37Haemorrhage not elsewhere classified arterial haemorrhage

BA00.Z Essential hypertension, unspecified Cै" arterial hypertension NOS :

BD52.4 Necrosis of artery $\equiv \mathbb{E}^{\circ}$

GC04.2 Ureteral fistula C Uretero-arterial fistula 8B01.0 Aneurysmal subarachnoid haemorrhage $G$ Vertebral artery aneurysm :

8B10 Transient ischaemic attack C" vertebral artery syndrome :

8B21 Asymptomatic stenosis of intracranial or extracranial artery $\mathrm{C}^{\mathrm{C}}$ stenosis of carotid artery NOS :

8B24.0 Dissection of cerebral arteries

8B24.Y Other specified cerebrovascular disease c

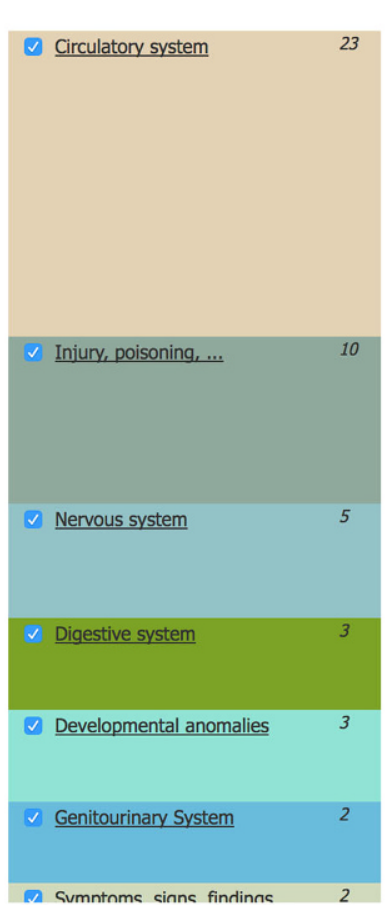

ICD-11 Coding Tool

arterial hypertension

Guessing the word being typed...

Word list

sort: Relatedness/repetition

hypertension

hypertensive
Destination Entities

BA00.Z Essential hypertension, unspecified c

arterial hypertension NOS :

BB01.0 Pulmonary arterial hypertension :

LD24.8Y Other specified acromelic dysplasias Cै Brachydactyly - arterial hypertension

8A84.Y Other specified secondary headache $G$

Headache attributed to arterial hypertension

BA04 Secondary hypertension C"

Arterial hypertension due to renal artery stenosis secondary to vasculitis

BB01.4 Pulmonary hypertension with multifactorial mechanism C

Pulmonary arterial hypertension associated with chronic haemolytic anaemia

4B00.00 Constitutional neutropenia C

Pulmonary arterial hypertension - leukopenia - atrial septal defect
Let us know if you couldn't find what you were looking for

Chapter distribution / filter

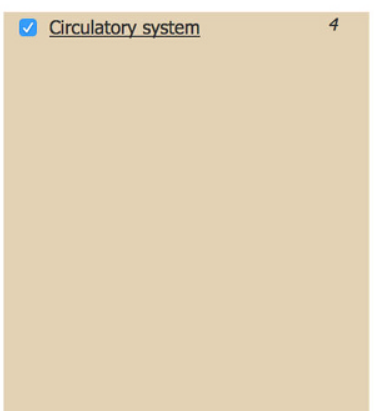

Abb. 1 ॥ Das Codierwerkzeug. Eine Wortliste und ein konfigurierbarer Kapitelfilter ermöglichen ein schnelles und korrektes Auffinden der gewünschten Schlüsselnummer. Die Suche nach dem Wort „arterial“ produziert automatisch eine Liste der assoziierten Wörter, eine Trefferliste und die Anzahl der Treffer auf Kapitelebene. Die Suche nach „arterial hypertension“ (arterieller Bluthochdruck) zeigt, wie bei korrekter Übereinstimmung des Suchbegriffes der Eintrag in der Ergebnisspalte gesondert in hellblau hervorgehoben wird 
gebungen mit sehr begrenzten Ressourcen und für psychische und Verhaltensstörungen in Vorbereitung. Andere Fassungen, wie für seltene Erkrankungen oder Dermatologie, sind in Planung.

\section{Inhaltliche Neuerungen im Vergleich zur ICD-10}

Die ICD-11 hat neben all den technischen Neuerungen auch eine inhaltliche Überarbeitung erfahren.

Die Spaltung des früheren Kapitels III der ICD-10 in „Erkrankungen des Immunsystems“ und "Erkrankungen des Blutes" erlaubt eine verbesserte Darstellung, unter anderem der Allergien [19, 20]. Auch finden sich im Kapitel zum Immunsystem systemische Immunerkrankungen, wie zum Beispiel die „nicht organspezifischen systemischen Autoimmunerkrankungen" (NOSSAD) des rheumatischen Formenkreises.

Ein neues Kapitel zu „Zuständen, die die sexuelle Gesundheit betreffen“, erlaubt die Klassifizierung entsprechender Zustände, wie z.B. die Gender-Inkongruenz ohne Verbindung zu somatischen oder geistigen Erkrankungen.

Das neue Kapitel „Erkrankungen des Schlafes“ gruppiert Gesundheitszustände, die vormals auf die Kapitel „Psychische und Verhaltensstörungen“, „Krankheiten des Nervensystems“ und „Krankheiten des Atmungssystems" der ICD-10 verteilt waren.

Im Kapitel „Symptome und abnorme klinische und Laborbefunde, die anderenorts nicht klassifiziert sind“, findet sich neben zahlreichen neuen Symptomen auch ein Abschnitt zur verbesserten Verschlüsselung von Antibiotikaresistenzen.

Das Kapitel „Traditionelle Medizin“ dient der Verschlüsselung von Diagnosen aus der traditionellen Medizin zusätzlich zur Codierung der Diagnose in „westlicher Medizin“.

Im Kapitel zu den äußeren Ursachen für Morbidität und Mortalität befindet sich eine komplett überarbeitete Sektion zur Dokumentation in Zusammenhang mit Patientensicherheit [21].

Eine große Neuerung ist auch das Kapitel „Extension codes“. Es enthält unter anderem Listen zur Anatomie, Laterali- tät, zu zeitlichem Verlauf, Schweregraden, Frakturtypen, Krankheitserregern, Chemikalien, Arzneistoffen (mit Bezug auf den internationalen Freinamen, International Nonproprietary Name INN) und auch Histopathologie. Diese können zur Verfeinerung der Beschreibung einer Diagnose bis auf das klinische oder in anderen Anwendungsfällen gewünschte Niveau verwendet werden. Histopathologie und Anatomie sind mit dem Kap. 2 „Neoplasien“ verbunden und werden zusammen zur Generierung der ICD-O-4 verwendet werden. Eine andere Gruppe von Extension Codes erlaubt die Angabe der Diagnosesicherheit oder von Art und Auftreten einer Diagnose (z. B. „Auftreten während des Aufenthaltes in einer Institution").

Im Codierbrowser finden sich Vorschläge für optionale Zusatzverschlüsselung wie auch Hinweise zur zwingend erforderlichen Verwendung von Schlüsselkombinationen.

Neben zahlreichen inhaltlichen Überarbeitungen in allen Kapiteln ist noch die ebenfalls neue Sektion „Funktionsfähigkeit“ zu erwähnen. Ein Satz von Kategorien, der der Internationalen Klassifikation der Funktionsfähigkeit (ICF) entnommen ist, erlaubt die Beschreibung der Funktionsfähigkeit einer Person in den Bereichen Aktivität und Teilhabe. Der Satz ist so gewählt, dass sowohl die WHO Disability Assessment Schedule 2 (WHODAS2) wie auch der WHO Model Disability Survey (MDS) zur Berechnung einer Maßzahl herangezogen werden kann. Auf diese Weise können Hilfsbedürftigkeit wie auch die Auswirkung von krankheitsbezogenen Verläufen auf den Grad der Funktionsfähigkeit bestimmt werden. Eine Anleitung zur Verwendung dieses Abschnittes, einschließlich eines Musterfragebogens, befindet sich gegenwärtig in Bearbeitung.

\section{Fazit}

- Die ICD ist in einem zehnjährigen offenen Prozess in allen Bereichen überarbeitet worden. Zahlreiche Anwendungszwecke werden nun abgedeckt. Die Möglichkeit, Schlüsselnummern zwecks detaillierterer Beschreibung einer Diagnose zu kombinieren, trägt neben der zeitgemäßen Darstellung und Gruppierung der Krankheiten zu einer erheblichen Verbesserung der klinischen Anwendbarkeit der ICD bei. Schlüsselkombinationen werden allerdings zukünftig auch in der Mortalitätsstatistik eine bessere Abbildung der Todesursachen ermöglichen. Das Regelwerk hierzu befindet sich gegenwärtig in Bearbeitung.

- Bei der Veröffentlichung der ICD-11 werden ein Implementierungspaket, bestehend aus dem Codiertool, Systematik, Handbuch Überleitungstabellen, Übersetzungsanleitung, und Informations- und Trainingsmaterial die Vorbereitung und Implementierung der ICD-11 vereinfachen.

- Im Rahmen der Arbeiten an der ICD-11 hat sich gezeigt, dass die klinischen Modifikationen der verschiedenen Länder in den meisten Bereichen ähnliche Zusätze in die ICD eingebaut haben - allerdings in leicht unterschiedlicher Art und Weise. Angesichts deren Einarbeitung in die ICD-11 zusammen mit den flexiblen Codiermöglichkeiten der ICD-11 stellt sich die Frage des Zusatznutzens zukünftiger nationaler Modifikationen der ICD.

- Alle Neuerungen und Funktionen der ICD-11 werden ausführlich im Referenzhandbuch der ICD-11 beschrieben, das in der jeweils aktuellen Fassung im Onlinebrowser zugänglich ist. Zusammen mit der neuen technischen Infrastruktur lässt sich sicher sagen, dass sich die ICD nun ins 21. Jahrhundert bewegt hat. Ein Pflegemechanismus wird die Aktualität der ICD auch zukünftig sicherstellen.

\section{Haftungsausschluss}

Der Autor ist Mitarbeiter der Weltgesundheitsorganisation. Der Autor ist alleine verantwortlich für die in diesem Artikel geäußerten Ansichten, die nicht unbedingt die Entscheidungen, Regelungen oder Ansichten der Weltgesundheitsorganisation darstellen. 


\section{Korrespondenzadresse}

\section{R. Jakob}

Weltgesundheitsorganisation (WHO)

Avenue Appia 20, 1211 Genf, Schweiz

jakobr@who.int

\section{Einhaltung ethischer Richtlinien}

Interessenkonflikt. R. Jakob gibt an, dass kein Interessenkonflikt besteht.

Dieser Beitrag beinhaltet keine von den Autoren durchgeführten Studien an Menschen oder Tieren.

Open Access Dieser Artikel wird unter der Creative Commons Namensnennung 4.0 International Lizenz (http://creativecommons.org/licenses/by/4.0/deed. de) veröffentlicht, welche die Nutzung, Vervielfältigung, Bearbeitung, Verbreitung und Wiedergabe in jeglichem Medium und Format erlaubt, sofern Sie den/die ursprünglichen Autor(en) und die Quelle ordnungsgemäßnennen, einen Link zur Creative Commons Lizenz beifügen und angeben, ob Änderungen vorgenommen wurden.

\section{Literatur}

1. WHO The Startup Mortality List (ICD-10-SMoL), (2016). http://www.who.int/healthinfo/civil registration/smol/en/.Zugegriffen: 14.02.2018

2. ICD (2017) ICD-10-CM - international classification of diseases, tenth revision, clinical modification. https://www.cdc.gov/nchs/icd/icd10 cm.htm (Erstellt: 1.Sept. 2017).Zugegriffen: 14. Febr. 2018

3. Jetté B et al (2010) The development, evolution, and modifications of ICD-10: challenges to the international comparability of morbidity data. Med Care 48(12):1105-1110

4. Üstün BT, Jakob RR (2005) Calling a spade a spade: meaningful definitions of health conditions. Bull World Health Organ 83:802-802

5. Tudorache T, Nyulas C, Noy CF, Redmond T, Musen MA (2011) iCAT: a collaborative authoring tool for ICD-11. Workshop Ontologies come of Age in the Semantic Web(OCAS2011) 10 th International Semantic Web Conference, Bonn, 24.10.2011, S72

6. Ustun TB, Jakob R (2009) The overall development of ICD-11. Psychiatr Diagn Chall Prospects 8:233

7. Bedirhan Üstün $T$, Jakob R, Çelik C, Lewalle $P$, Kostanjsek N, Renahan M et al (2007) http:// www.who.int/classifications/icd/ICDRevision.pdf. Zugegriffen: 26.04.2018

8. Aymé S, Bellet B, Rath A (2015) Rare diseases in ICD11: making rare diseases visible in health information systems through appropriate coding. Orphanet J Rare Dis 10:35

9. WHO (2018) External review of ICD 11 revision. http://www.who.int/classifications/icd/ externalreview/en/.Zugegriffen: 14. Febr. 2018

10. WHO (2017) ICD-11 revision. http://www.who.int/ classifications/icd/revision/en/. Zugegriffen: 24. Apr. 2018

11. ICD ICD-11 beta draft-mortality and morbidity statistics (2018) https://icd.who.int/dev11/l-m/ en. Zugegriffen: 14.02 .2018

12. ICD ICD-11 coding tool (2018) https://icd.who.int/ devct11/icd11_mms/en/current\#/. Zugegriffen: 14.02.2018
13. ICD ICD-11 reference guide. (2018) https://icd. who.int/browse11.Zugegriffen: 26.04.2018

14. Schulz S, Rector A, Rodrigues JM, Spackman K (2012) Competing interpretations of disorder codes in SNOMED CT and ICD. AMIA Annual Symposium Proceedings, Bd. 2012, S819

15. Rodrigues JM et al (2014) ICD-11 and SNOMED CT common ontology: circulatory system. In: MIE, S 1043-1047

16. Chute C, Üstün B, Spackman K (2012) Ontologybased convergence of medical terminologies: SNOMEDCT and ICD-11.In:Proc. EHealth, S10-11

17. SNOMED CT to ICD-10-CM Map. [Online]. (2018) https://www.nlm.nih.gov/research/umls/ mapping_projects/snomedct_to_icd $10 \mathrm{~cm} . \mathrm{html}$. Zugegriffen:03.02.2018

18. Schulz S et al (2014) What's in a class? Lessons learnt from the ICD-SNOMED CT harmonisation. Stud Health Technol Inform 205:1038-1042

19. Tanno LK, Chalmers RJG, Calderon MA, Aymé $S$, Demoly $\mathrm{P}$, on behalf the Joint Allergy Academies (2017) Reaching multidisciplinary consensus on classification of anaphylaxis for the eleventh revision of the World Health Organization's (WHO) International Classification of Diseases (ICD-11). Orphanet J Rare Dis 12(1):53

20. Tanno LK et al (2015) Constructing a classification of hypersensitivity/allergic diseases for ICD-11 by crowdsourcing the allergist community. Allergy 70(6):609-615

21. GhaliWA (2013) ICD-11 for quality and safety: overview of the WHO quality and safety topic advisory group. Int J Qual Health Care 25(6):621-625 